\title{
Eigenenergies of a Nanoscale Symmetric Double Triangular Quantum Well in Double Gate InAlAs/InGaAs HEMT
}

\author{
Neha Verma, Jyotika Jogi \\ Microelectronics Research Laboratory, Department of \\ Electronic Science \\ A.R.S.D College, University of Delhi South Campus \\ New Delhi 110021, India \\ jogijyotika@rediffmail.com
}

\author{
Enakshi Khular Sharma \\ Department of Electronic Science \\ University of Delhi South Campus \\ New Delhi 110021, India \\ enakshi54@yahoo.co.in
}

\begin{abstract}
This paper presents the analytical calculation of the eigenenergies for a nanoscale symmetric double triangular quantum well (DTQW) in double gate InAIAs/InGaAs HEMT. The symmetric double triangular quantum wells formed by two similar heterostructures are separated by a barrier in the InGaAs channel. The paper accounts for the quantization effects by solving a one-dimensional (1D) time-independent Schrodinger equation in the nanoscale channel at equilibrium i.e. when no gate voltage is applied. In particular, the ground and first excited energy levels are numerically calculated which represent the lowest populated sub-bands.
\end{abstract}

Keywords- double gate HEMT; double triangular quantum well (DTQW); eigenenergies; symmetric; Schrodinger equation

\section{INTRODUCTION}

The modulation doped field effect transistors (MODFETs) or High electron mobility transistors (HEMTs) replaced MESFET as the leading microwave FET [1] due to low noise and high power at millimeter-wave frequencies $(30-300 \mathrm{GHz})$ [2]. Presently, DG-HEMTs (double gate HEMTs) are widely accepted due to their larger 2-DEG (two-dimensional electron gas) concentration and higher transconductance relative to a single channel HEMT [3]. As attempts are made at scaling down the device to nano-dimensions, there is a requirement to understand and study the quantum effects in the device.

Quantum effects play significant role in structures where the size is of the order of $100 \mathrm{~nm}$ or less. The carriers in this quantum regime can now be represented as an electron wave and its interaction with the device boundaries leads to the quantization effects. In the present work, authors considered the quantum effects in a nanoscale symmetric InAlAs/InGaAs DG-HEMT. The device has a symmetric double heterostructure forming two identical 2-DEGs (two dimensional electron gas) in the channel. The discontinuities on both the conduction and valence band edges occur at the heterointerface forming a potential well causing the confinement of carriers inside the well. The quantum mechanical system comprises of two symmetric double triangular quantum wells separated by a barrier. The eigenenergies corresponding to symmetric and antisymmetric states are calculated by solving a one-dimensional
(1D) time-independent Schrödinger equation for the symmetric DTQW potential profile separated by a barrier under equilibrium with zero gate bias. The resulting transcendental equation is solved numerically to determine the eigenvalues.

\section{MODEL}

This paper deals with the InAlAs/InGaAs DG-HEMT with gate length $100 \mathrm{~nm}$ which has been fabricated using transferred substrate technique by Wichmann et al. [4]. The schematic of the device is shown in Fig. 1. The carriers are presumed to be confined inside the two quantum wells formed in the channel. The energy-band diagram for a double heterostructure with double quantum wells formed in z-direction (perpendicular to the channel) is presented in Fig. 2.

The potential profile, $V(\mathrm{z})$, for the configuration in equilibrium is shown in Fig. 3. The origin of the coordinates is chosen at the centre of the barrier. The channel thickness of the device is taken as $20 \mathrm{~nm}$. The barrier thickness and the width of each well are taken as $2 a$ where $a$ is one sixth of the channel width. $V_{o}$ representing the height of the barrier (conduction band discontinuity) for symmetric $\mathrm{In}_{0.52} \mathrm{Al}_{0.48} \mathrm{As} / \operatorname{In}_{0.53} \mathrm{Ga}_{0.47} \mathrm{As}$ DG-HEMT as $0.5 \mathrm{eV}$.

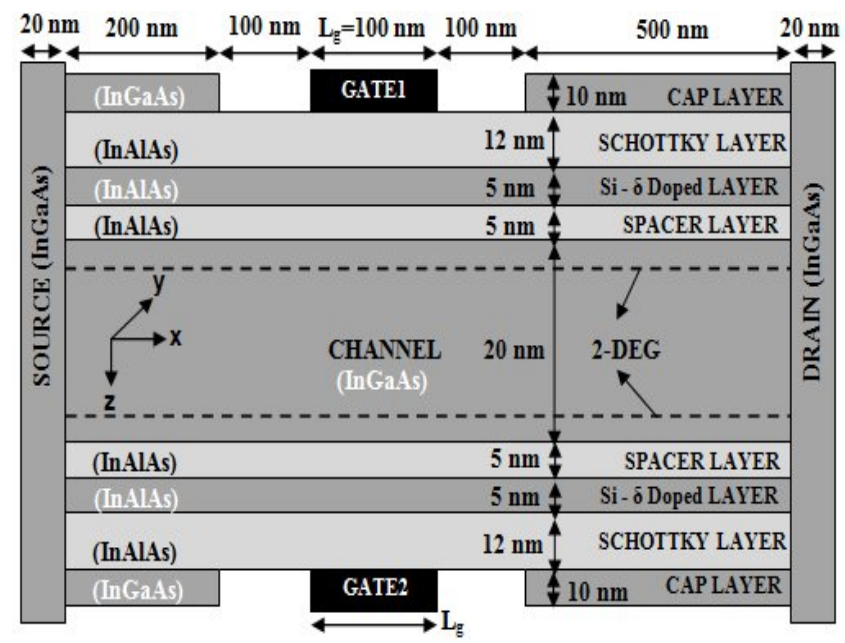

Figure 1. Structure of DG-HEMT. 




Figure 2. Energy Band diagram for double heterostructure DG-HEMT.

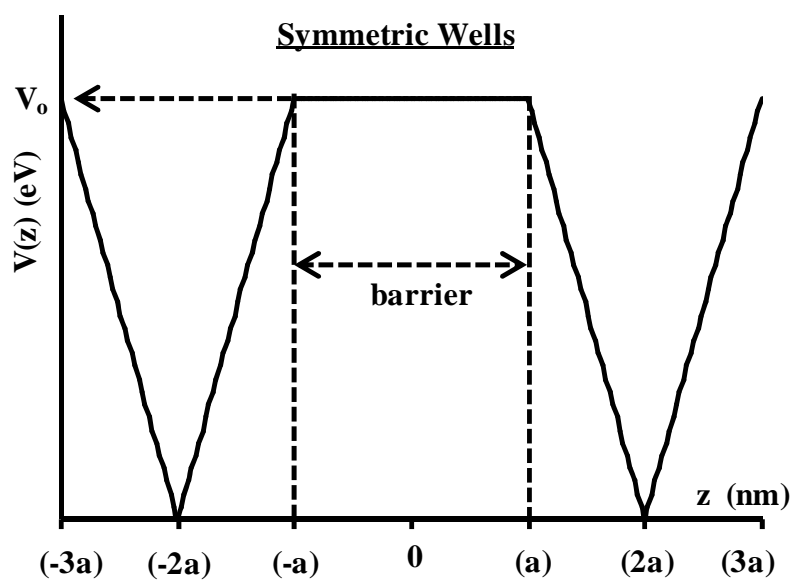

Figure 3. Potential Profile of a symmetric DTQW separated by a barrier formed in the channel.

Thus potential profile for DTQW separated by a barrier with its boundary regions is characterized as following:

$$
V(z)=\left\{\begin{array}{l}
V_{O} \cdot\left(\frac{-2 a-z}{a}\right), z \leq-2 a, \\
V_{O} \cdot\left(\frac{z+2 a}{a}\right),-2 a \leq z \leq-a, \\
V_{O},|z| \leq a, \\
V_{O} \cdot\left(\frac{2 a-z}{a}\right), a \leq z \leq 2 a, \\
V_{O} \cdot\left(\frac{z-2 a}{a}\right), z \geq 2 a
\end{array}\right.
$$

Since the potential profile is symmetric about the $z=0$ axis, it is sufficient to consider only the region $0 \leq z \leq 3 a$. The Schrodinger equation for the various regions can be written as follows:

For region $0 \leq z \leq a$ :

$$
\frac{d^{2} \psi(z)}{d z^{2}}-k^{2} \psi(z)=0
$$

where

$$
k^{2}=\frac{2 m^{*}}{\hbar^{2}} \cdot[V(z)-E]
$$

$E$ is the energy eigenvalue, $\hbar$ is the reduced Planck constant, $m^{*}$ is the effective mass of the electron, $\psi(z)$ is the wavefunction and $V(z)$ is defined in (1).

For region $a \leq z \leq 2 a$ and $z \geq 2 a$ :

$$
\frac{d^{2} \psi}{d \xi^{2}}-\xi \psi=0
$$

where the solutions of (4) are the Airy functions [5] with $\xi$ as their argument.

The wave function $\psi(z)$ for $0 \leq z \leq 3 a$ region for the symmetric state will have a solution as follows:

$$
\psi(z)=\left\{\begin{array}{l}
C_{1} \cdot \cosh (k z), 0 \leq z \leq a \\
C_{2} \cdot \operatorname{Ai}\left(\xi_{2}\right)+D_{2} \cdot \operatorname{Bi}\left(\xi_{2}\right), a \leq z \leq 2 a \\
C_{3} \cdot \operatorname{Ai}\left(\xi_{3}\right), z \geq 2 a
\end{array}\right.
$$

where $\mathrm{Ai}$ and $\mathrm{Bi}$ are the regular and the irregular Airy functions and their arguments are defined as:

$$
\xi_{2}=\left(\frac{V_{o} \cdot \pi^{2}}{\left(\hbar^{2} \pi^{2} / 2 m^{*} a^{2}\right)}\right)^{1 / 3} \cdot\left[\left(\frac{2 a-z}{a}\right)-\frac{E}{V_{o}}\right]
$$

and

$$
\xi_{3}=\left(\frac{V_{o} \cdot \pi^{2}}{\left(\hbar^{2} \pi^{2} / 2 m^{*} a^{2}\right)}\right)^{1 / 3} \cdot\left[\left(\frac{z-2 a}{a}\right)-\frac{E}{V_{o}}\right]
$$

The unknown coefficients $\mathrm{C}_{1}, \mathrm{C}_{2}, \mathrm{D}_{2}$ and $\mathrm{C}_{3}$ are obtained by applying the boundary conditions at $\mathrm{z}=a$ and $\mathrm{z}=2 a$, which leads to a $4 \times 4$ determinant equation or eigenvalue equation as (8). This is solved numerically to obtain the eigenenergies for the symmetric states.

$$
\left|\begin{array}{cccc}
\cosh (k a) & -A i\left(\xi_{2}{ }^{a}\right) & -B i\left(\xi_{2}{ }^{a}\right) & 0 \\
k \cdot \sinh (k a) & -\chi_{a} \cdot A i^{\prime}\left(\xi_{2}{ }^{a}\right) & -\chi_{a} \cdot B i^{\prime}\left(\xi_{2}{ }^{a}\right) & 0 \\
0 & A i\left(\xi_{2}{ }^{2 a}\right) & B i\left(\xi_{2}{ }^{2 a}\right) & -A i\left(\xi_{3}{ }^{2 a}\right) \\
0 & -A i^{\prime}\left(\xi_{2}{ }^{2 a}\right) & -B i^{\prime}\left(\xi_{2}{ }^{2 a}\right) & -A i^{\prime}\left(\xi_{3}{ }^{2 a}\right)
\end{array}\right|=0
$$


where

$$
\begin{aligned}
\xi_{2}{ }^{a} \text { is }\left.\xi_{2}\right|_{z=a}, & \\
\xi_{2}{ }^{2 a}, \xi_{3}{ }^{2 a} \text { are }\left.\xi_{i}\right|_{z=2 a} \text { for } i=2,3 \text { and } & \\
\chi_{a} & =\left[\left(\frac{V_{o} \cdot \pi^{2}}{\left(\hbar^{2} \pi^{2} / 2 m^{*} a^{2}\right.}\right)^{1 / 3} \cdot\left(-\frac{1}{a}\right)\right]
\end{aligned}
$$

A similar treatment was followed for the antisymmetric states, to obtain the corresponding eigenenergies.

\section{RESULTS}

The various parameters used in the calculation are as follows: effective mass $m^{*}=0.033^{*} \mathrm{~m}_{0}$, where $\mathrm{m}_{0}$ is the electron rest mass, quantum well and barrier thickness $(6.6$ $\mathrm{nm})$ and barrier height $V_{o}=0.5 \mathrm{eV}$. The eigenenergies obtained for the ground and first excited state corresponding to the symmetric and antisymmetric state are presented in Fig. 4 and the values are:

$\mathrm{E}_{0}($ symmetric $)=0.2956(\mathrm{eV})$ and

$\mathrm{E}_{1}($ antisymmetric $)=0.3006(\mathrm{eV})$.

The two energy levels are found to be very close to each other i.e. $\mathrm{E}_{0} \approx \mathrm{E}_{1}$.

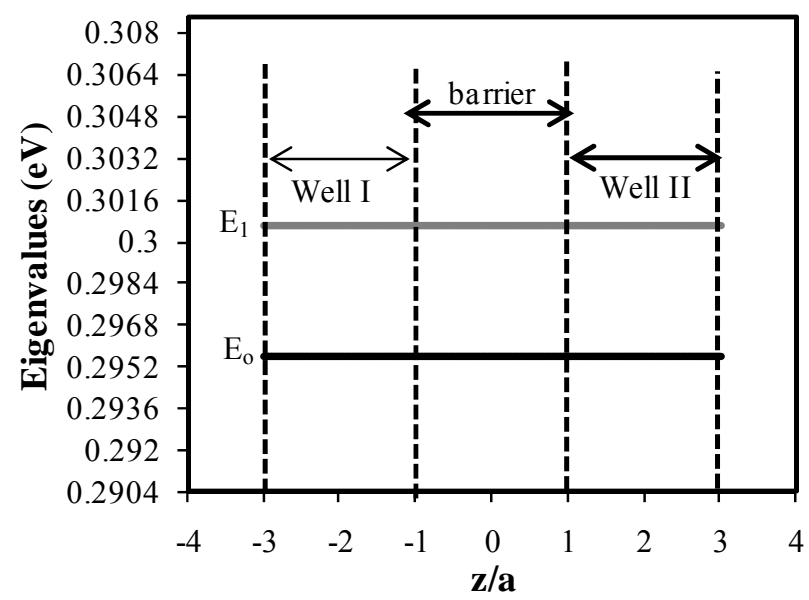

Figure 4. Lowest two eigenenergies $\left(\mathrm{E}_{0}\right.$ and $\left.\mathrm{E}_{1}\right)$ with their corresponding eigenvalues.

\section{CONCLUSION}

This paper deals with the quantum effects in the nanoscale channel of symmetric InAlAs/InGaAs DG-HEMT by solving a one-dimensional (1D) time-independent Schrodinger equation for a symmetric DTQW potential profile separated by a barrier at equilibrium. The lowest eigenenergies corresponding to symmetric and antisymmetric states are calculated by solving eigenvalue equation numerically. The lowest two values of energy levels are found close to each other implying that under equilibrium condition the splitting of the energy states is insignificant and hence a shift of carriers from one well to the other will not take place under the given conditions at room temperature. Future scope of this work would include studying the behavior of wave functions corresponding to the eigensates in the channel under equilibrium and under the influence of applied gate bias.

\section{ACKNOWLEDGMENT}

The authors acknowledge University Grants Commission for providing financial support for this work.

\section{REFERENCES}

[1] H. Morkoc, H. Unlu and G. Ji, Principles and Technology of MODFETs, vol. 2, England: John Wiley \& Sons Ltd., 1991, ch. 7.

[2] P. C. Chao, S. C. Palmateer, P. M. Smith, U. K. Mishra, K. H. G Duh, and J. C. M Hwang, "Millimeter-wave low-noise high electron mobility trabsistors," IEEE Electron Dev Lett, vol. EDL-6, no. 10, pp. 531-533, October 1985.

[3] B. G. Vasallo, N. Wichmann, S. Bollaert, A. Cappy, T. Gonzalez, D. Pardo and J. Mateos, "Monte Carlo Comparison Between InP-Based Double-Gate and Standard HEMTs," Proc. EMICC Conf. 2006, pp. 304-307, September 2006.

[4] N. Wichmann, I. Duszynski, S. Bollaert, J. Mateos, X. Wallart and A. Cappy, "100nm InAlAs/InGaAs Double-Gate HEMT using transferred substrate," IEEE IEDM Conf. 2004, IEDM Technical Digest, pp. 1023-1026, December 2004.

[5] O. Vallee and M. Soares, Airy Functions and Applications to Physics, London: World Scientific, Imperial College press, 2004. 